\title{
Two-wavelength switching with a distributed- feedback semiconductor optical amplifier (DFBSOA)
}

\author{
A. Hurtado, A. Gonzalez-Marcos, J.A. Martin-Pereda and M.J. Adams
}

\begin{abstract}
Switching of a signal beam by another control beam at different wavelength is demonstrated experimentally using the optical bistability occurring in a $1.55 \mu \mathrm{m}$-distributed feedback semiconductor optical amplifier (DFBSOA) working in reflection. Counterclockwise (S-shaped) and reverse (clockwise) bistability are observed in the output of the control and the signal beam respectively, as the power of the input control signal is increased. With this technique an optical signal can be set in either of the optical input wavelengths by appropriate choice of the powers of the input signals. The switching properties of the DFBSOA are studied experimentally as the applied bias current is increased from below to above threshold and for different levels of optical power in the signal beam and different wavelength detunings between both input signals. Higher on-off extinction ratios, wider bistable loops and lower input power requirements for switching are obtained when the DFBSOA is operated slightly above its threshold value.
\end{abstract}

\section{Introduction}

Optical bistability in laser diode amplifiers has attracted attention for use in optical communications and optical signal processing because of inherent advantages, including optical gain, low power requirements for operation, large fanout etc (for a review see [1]). Among others, the bistable properties exhibited by laser diode amplifiers have been used for the development of optical logic gates [2-4], flip-flops [5-7], optical signal regeneration [8, 9], wavelength conversion $[9,10]$ etc. Together with these applications, semiconductor laser amplifiers have also attracted attention for their potential use to perform optical switching between two simultaneously injected optical signals and its application in optical telecommunication networks.

The first studies of bistable switching of two signals at different wavelength were carried out based on the optical bistability occurring in a Fabry-Perot semiconductor laser amplifier (FPSLA) working in transmission. Kawaguchi et al. [11] were the first to report the appearance of anticlockwise and reverse optical bistability when two optical signals at different wavelengths were injected into an FPSLA biased below threshold. Based on that scheme, Inoue [12] reported the switching of two signals at different wavelengths with a bit rate of $800 \mathrm{Mb} / \mathrm{s}$. Okada et al. [13] studied both theoretically and experimentally the optical bistability in an FPSLA biased slightly above threshold

\footnotetext{
A Hurtado is with the Department of Electronic Systems Engineering, University of Essex, Colchester $\mathrm{CO} 43 \mathrm{SQ}$, UK, on leave from the Department of Photonic Technology, Universidad Politecnica de Madrid, Spain A. Gonzalez-Marcos and J.A. Martin-Pereda are with the Department of Photonic Technology, Universidad Politecnica de Madrid, Spain

M.J. Adams if with the Department of Electronic Systems Engineering, University of Essex, Colchester CO4 3SQ, UK

E-mail: hurtado@tfo.upm.es
}

with two injected input signals at different wavelengths and demonstrated set-reset operation. Grosskopf et al. [14] demonstrated an optically controlled switch with an AR-coated $0.78 \mu \mathrm{m}$ GaAlAs semiconductor laser amplifier with bit rates up to $100 \mathrm{MHz}$. More recently attention has focused on distributed feedback semiconductor optical amplifiers (DFBSOAs). Maywar et al. demonstrated 1.3-1.55 $\mu \mathrm{m}$ wavelength conversion [15] and optical flipflop operation [16] under simultaneous injection of two optical signals at different wavelengths into a DFBSOA working in transmission.

In this paper experimental two-wavelength switching based on the dispersive optical bistability occurring in a $1.55 \mu \mathrm{m}$ DFBSOA operated in reflection is reported. Switching of one signal beam by a control beam at a different wavelength is reported when the DFBSOA is operated with a bias current either below or above the threshold value. The device switching properties are studied in terms of the injected optical power and the wavelength difference between the input and control signal beams. Also, the evolution of the switching properties of the device are studied when the applied bias current varies gradually from below to above the threshold of the DFBSOA.

\section{Bistability and switching}

The optical bistability is characterised by a dispersive nonlinearity, where the refractive index of the medium in the active region of the device is dependent on the amount of injected power. Under external optical power injection, owing to the change in the refractive index and the saturation of gain, the resonant cavity wavelength of the laser amplifier moves towards longer wavelengths depending on the amount of the injected power, and exceeding a threshold value the optical bistability can be observed $[17$, $18]$. In the past, the dispersive optical bistability has been studied in FPSLAs [17-21] in DFBSOAs [22-24] and more recently in vertical-cavity semiconductor optical amplifiers (VCSOAs) $[25,26]$. The optical bistability has 
been analysed when the amplifier is operated either in transmission or in reflection, and the differences in behaviour for each mode of operation have been determined [21, 27].

To obtain two-wavelength switching with a DFBSOA, the wavelength of the input control signal $\left(\lambda_{1}\right)$ is chosen to be detuned to the long wavelength side of resonant peak of the DFBSOA, while the wavelength of the input signal beam $\left(\lambda_{2}\right)$ is set at that of the resonance of the DFBSOA $\left(\lambda_{\mathrm{DFBSOA}}\right)$. As the wavelength of the input signal beam coincides with the resonant wavelength of the DFBSOA, this signal will exhibit optical gain at the output of the DFBSOA. Initially, the power of the input control signal $\left(P_{i n 1}\right)$ and the power of the input signal beam $\left(P_{i n 2}\right)$ are both low enough that the gain is unsaturated. Increasing the power of the input control signal will produce a decrease of the carrier concentration due to stimulated recombination processes, which will cause an increase of the refractive index in the active region. As a consequence the resonant peak of the DFBSOA will shift towards longer wavelengths and the output of the control signal will grow linearly. The output of the signal beam decreases linearly as the resonance wavelength moves away towards longer wavelengths. At a certain point, the resonance of the DFBSOA will match the wavelength of the control signal, and the output of the control signal will switch abruptly from an 'off' to an 'on' state, while in the output of the signal beam the reverse phenomenon will happen, abruptly switching from an 'on' to an 'off' state. Finally, if the input power of the control signal is now decreased the reverse process occurs with hysteresis. Consequently, anticlockwise bistability is obtained in the output/input power characteristic of the control signal $\left(P_{\text {out } 1}, P_{i n 1}\right)$, while clockwise or reverse bistability is obtained in the output signal versus input control signal transfer function $\left(P_{\text {out } 2}, P_{i n 1}\right)$. This complimentary process might be used for the switching of an optical signal by another one at a different wavelength.

\section{Experimental setup}

The switching between two optical signals at different wavelengths with a DFBSOA has been studied with the experimental arrangement shown in Fig. 1. An all-fibre system has been used to launch light from two laser sources into the DFBSOA. The source for the input control beam $\left(P_{m n 1}, \lambda_{1}\right)$ is an external cavity semiconductor laser whose emitting wavelength could be continuously tuned over a spectral range of $1500-1570 \mathrm{~nm}$ by rotating a grating. The source used for the input signal beam $\left(P_{i n 2}\right.$, $\lambda_{2}$ ) is a commercial $1.55 \mu \mathrm{m}$ DFB laser. The emission wavelength of the DFB laser source is tuned by changing the heat-sink temperature. An optical isolator is used after

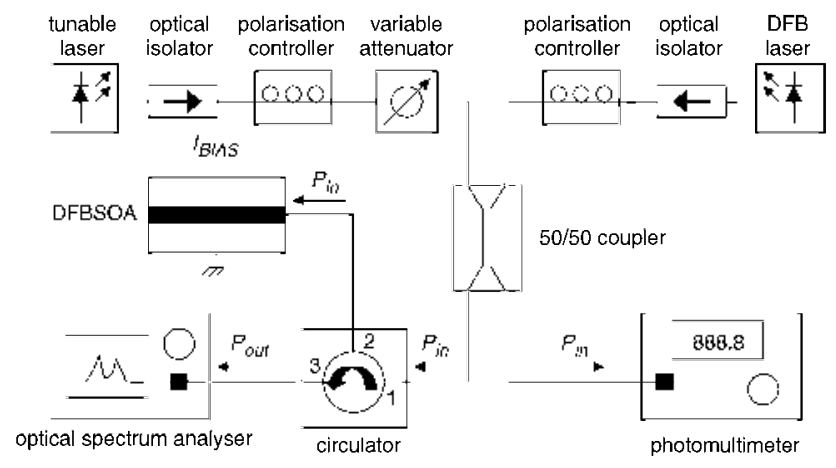

Fig. 1 Experimental setup for two-wavelength switching measurements both laser sources in order to avoid backward reflections that could lead to spurious results. The DFBSOA was a commercial $1557 \mathrm{~nm}$ DFB laser with a threshold current of $16.4 \mathrm{~mA}$ at $298 \mathrm{~K}$. The temperature of the DFBSOA was kept constant for all experiments at $298 \mathrm{~K}$. Two fibre birefringence polarisation controllers were included in the setup to match the polarisation state between the DFBSOA and the light emitted by the two laser sources. A variable attenuator was used to vary the optical input power of the signal beam. The outputs from both laser sources are combined into a single fibre using a directional coupler. The second output of the coupler is connected to a photomultimeter to monitor the optical input power. Finally, an optical circulator was included in the experimental setup to inject the combined optical signals into the amplifier and to monitor the reflected signal of the DFBSOA. The measurements of the reflected signal were taken with an optical spectrum analyser.

\section{Results}

Two-wavelength switching based in the optical reflective bistability occurring in a DFBSOA is reported when injecting two optical signals at different wavelengths into the amplifier. The evolution in the two-wavelength switching as the applied bias current is increased from below to above threshold has been analysed. Finally, the influence of the power of the input signal beam and the wavelength difference between input signals on the switching properties of the DFBSOA have also been studied for both below and above threshold.

\subsection{Influence of the bias current}

The bistable switching between two signals at different wavelengths has been studied for different bias currents applied to the DFBSOA. The evolution of the switching properties is studied as the DFBSOA is biased from below to above its threshold current, in an interval ranging from $15.5 \mathrm{~mA}\left(0.945 I_{t h}\right)$ to $17.25 \mathrm{~mA}\left(1.05 I_{i h}\right)$. It should be noted that the gain saturation caused by injection of optical signals into a DFBSOA may cause some increase of the threshold current. To investigate this effect, Fig. $2 a$ shows a series of measured light-current characteristics (normalised to threshold) for different input optical powers. The corresponding change of normalised threshold current with input power shown in Fig. $2 b$ indicates a maximum increase of $5 \%$ for the highest power level used here $(200 \mu \mathrm{W})$. To have a unique reference current for the switching measurements, the value of threshold current in the absence of external optical injection is used here. Figures $3 a$ and $3 b$ show, respectively, (against the input control signal, $P_{m n 1}$ ), the experimental I/O power relations of the output control signal $\left(P_{\text {out } 1}, P_{i n 1}\right)$ and output signal beam $\left(P_{\text {out } 2}, P_{\text {in } 1}\right)$. The input signal beam $\left(P_{i n 2}\right)$ is set with a constant power of $50 \mu \mathrm{W}$ at the wavelength of the resonant peak of the DFBSOA and the input control is detuned $0.25 \mathrm{~nm}$ to its long wavelength side. It was found for the measurements reported here that no nonlinear interactions between the external optical signals and the lasing mode of the DFBSOA were observed. Finally, the main experimental results observed as the bias current applied to the DFBSOA varies gradually from below to above its threshold level, are collected in Table 1.

Figures $3 a$ and $3 b$ show that the observed hysteresis cycles are wider when the applied current is higher than the threshold value. This behaviour is exactly the same as that reported in the study of the optical bistability in a 

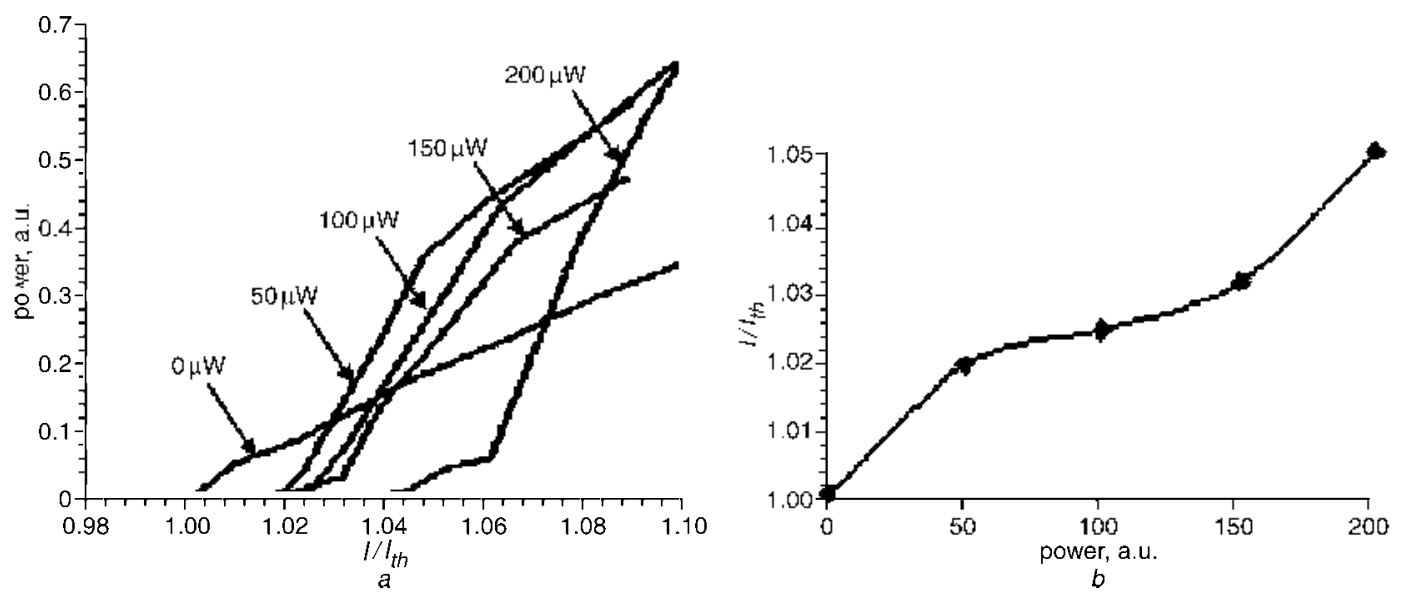

Fig. 2 Change in the threshold current of the DFBSOA with external optical injection

a L-I curves for different external optical power injection levels

$b$ Relative change in the bias current as a function of external power

laser amplifier with a single input beam [28]. Figures $3 a$ and $3 b$ also show that, either below or above threshold, as the bias current applied to the DFBSOA is increased, the hysteresis cycles associated with bistable switching become wider in both cases. But the evolution in the input control signal power requirements $\left(P_{i n 1}\right)$ are completely different in the cases below and above threshold. Below threshold, an increase in the applied bias current results in the reduction of the optical power of the input control signal needed for the achievement of wavelength switching. In the above threshold case, when increasing the bias current, the power requirements for the appearance of
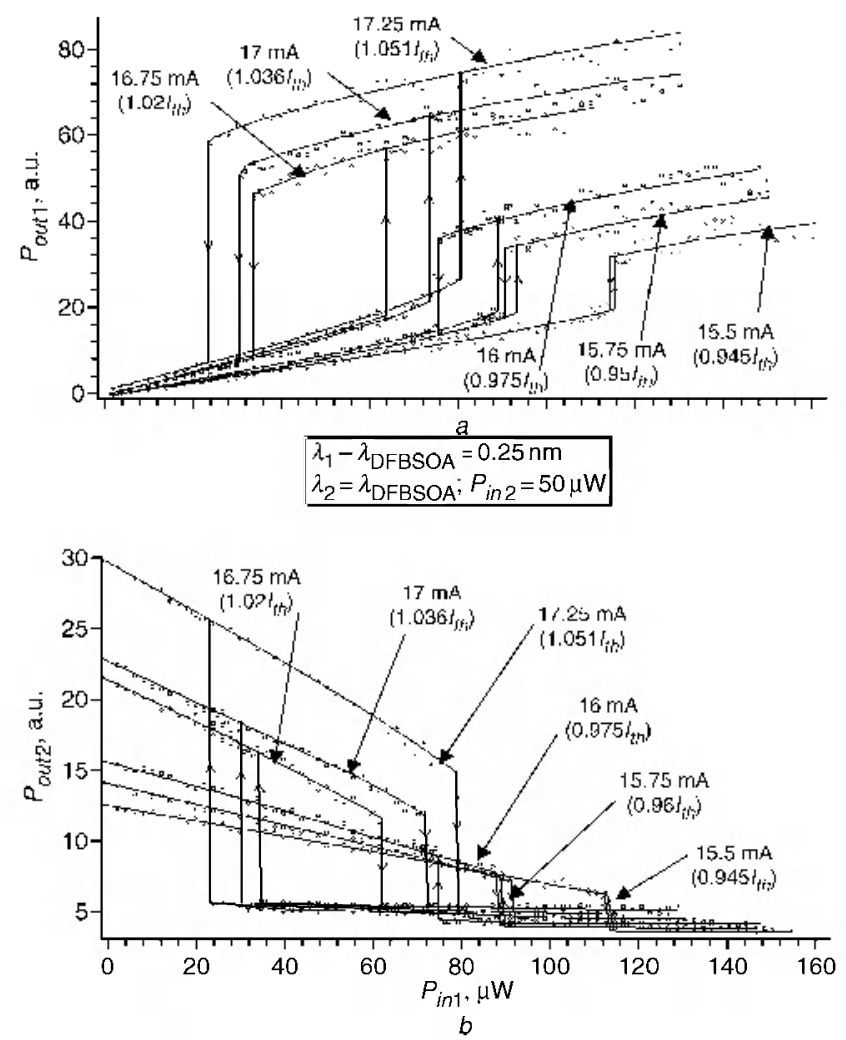

Fig. 3 Study of the two-wavelength switching when the bias current applied to the DFBSOA is increased from below to above threshold as indicated

a $\mathrm{I} / \mathrm{O}$ power characteristic of the control signal showing counterclockwise bistability

$b$ Output signal versus input control showing reverse bistability switching are increased as well. This observed evolution has the same behaviour as that observed when studying the optical bistability in a semiconductor laser amplifier with only one input beam $[20,21]$.

It is also remarkable, as can be seen in Table 2 and Fig. 4, that the power requirements needed for the achievement of bistable switching are higher below than slightly above threshold for the range of currents applied in the experiment. The minimum power requirements for switching are obtained for an applied bias current approximately equal to the threshold value, but as soon as the bias current is increased further above threshold, the power needed for switching grows gradually, and exceeds the levels observed in the below threshold case.

\subsection{Influence of the power of the input signal beam}

Figures $5(a-d)$ show the measured $\mathrm{I} / \mathrm{O}$ power characteristics for the control $\left(P_{\text {out } 1}\right)$ and the signal beam $\left(P_{\text {out } 2}\right)$ versus the control input signal $\left(P_{m 1}\right)$. The experiments were carried out for different constant optical power levels of the input signal beam $\left(P_{i n 2}\right)$. The influence that the power of the input signal has on the bistable switching has been studied both when the DFBSOA operates below threshold with a current of $16 \mathrm{~mA}\left(97.5 I_{t h}\right.$ ) (see Figs. $5 a$ and $5 b$ ) and when the DFBSOA operates above its threshold level with a current of $17 \mathrm{~mA}\left(1.03 I_{t h}\right)$ (see Figs. $5 c$ and $5 d$ ). In all cases, the input signal beam was set at the wavelength of the resonant peak of the DFBSOA while the input control signal was detuned $0.25 \mathrm{~nm}$ to the long-wavelength side of the resonant peak of the amplifier. For the present experiment the constant power of the input signal beam has been varied from 25 to $200 \mu \mathrm{W}$. Finally, in Table 2 are listed the main experimental results measured when the power of the input signal $\left(P_{i n 2}\right)$ is modified.

In both cases, below and above threshold, as shown in Figs. $5(a-d)$ the higher the power of the input signal beam the lower the optical power needed in the control signal $\left(P_{i n 1}\right)$ for the achievement of bistable switching. At the same time, the hysteresis cycles become narrower as the input signal power $\left(P_{i n 2}\right)$ is increased and almost disappear when the DFBSOA works below threshold with an input signal power of $150 \mu \mathrm{W}$. To explain this dependence let us first recall that the external injection of an input signal beam to the DFBSOA reduces the carrier concentration in 
Table 1: Influence of the applied bias current in the two-wavelength switching

\begin{tabular}{|c|c|c|c|c|c|c|}
\hline Bias current $(\mathrm{mA})$ & 15.50 & 15.75 & 16.00 & 16.75 & 17.00 & 17.25 \\
\hline Switch-up power $\lambda_{1}(\mu W)$ & 116 & 94 & 89 & 63 & 73 & 81 \\
\hline Switch-down power $\lambda_{1}(\mu W)$ & 115 & 90 & 75 & 33 & 30 & 23 \\
\hline Hysteresis cycle $(\mu W)$ & 1 & 4 & 14 & 30 & 43 & 58 \\
\hline Minimum on-off ratio $\lambda_{1}$ & 1.46 & 1.94 & 2.05 & 2.89 & 2.97 & 2.71 \\
\hline Minimum on-off ratio $\lambda_{2}$ & 1.61 & 1.72 & 1.70 & 2.09 & 2.22 & 2.36 \\
\hline
\end{tabular}

The switching between states occur at equal power levels both in $\lambda_{1}$ and $\lambda_{2}$

Table 2: Study of the influence of the input signal's power $\left(P_{I m 1}\right)$ in the two-wavelength switching with a DFBSOA

\begin{tabular}{|c|c|c|c|c|c|c|c|c|}
\hline Bias current $(\mathrm{mA})$ & 16 & & & & 17 & & & \\
\hline Input power $(\mu W)$ & 25 & 50 & 100 & 150 & 25 & 50 & 100 & 150 \\
\hline Switch-up power $\lambda_{1}(\mu W)$ & 97 & 89 & 77 & 77 & 80 & 73 & 58 & 46 \\
\hline Switch-down power $\lambda_{1}(\mu W)$ & 79 & 75 & 73 & 73 & 29 & 30 & 30 & 26 \\
\hline Hysteresis cycle width $(\mu \mathrm{W})$ & 18 & 14 & 4 & 4 & 51 & 43 & 28 & 20 \\
\hline Minimum on-off ratio $\lambda_{1}$ & 2.35 & 2.05 & 1.97 & 1.97 & 3.04 & 2.97 & 3.05 & 2.95 \\
\hline Minimum on-off ratio $\lambda_{2}$ & 1.88 & 1.70 & 1.43 & 1.43 & 2.63 & 2.22 & 1.78 & 1.49 \\
\hline
\end{tabular}

the active region due to stimulated recombination processes. This reduction is proportional to the total amount of injected power, the higher the injected power the lower is the carrier concentration in the active region. As a result, the refractive index of the active region is increased and the saturation of gain, that causes the bistable switching process, is attained for lower power levels of the input control signal $\left(P_{t n 1}\right)$. Consequently, continuously increasing the power of the input signal beam makes the bistable switching appear for lower input control signal power levels and with a reduced hysteresis cycle width.

\subsection{Influence of the relative wavelength difference}

The influence of the wavelength difference between the input control and input signal beam on the bistable switching between both signals is also analysed. The influence of this parameter is once again studied for the below and above threshold regimes. In all cases the input signal beam is configured with a constant power level of $50 \mu \mathrm{W}$.

Two different cases are considered. Firstly, Figs. $6(a-d)$ show the power relationships between the outputs of the control $\left(P_{o u t 1}, P_{t n 1}\right)$ and the signal beam $\left(P_{o u t 2}, P_{i n 1}\right)$ versus the input control signal $\left(P_{m 1}\right)$. The wavelength of the input signal beam is set at the resonant peak of the

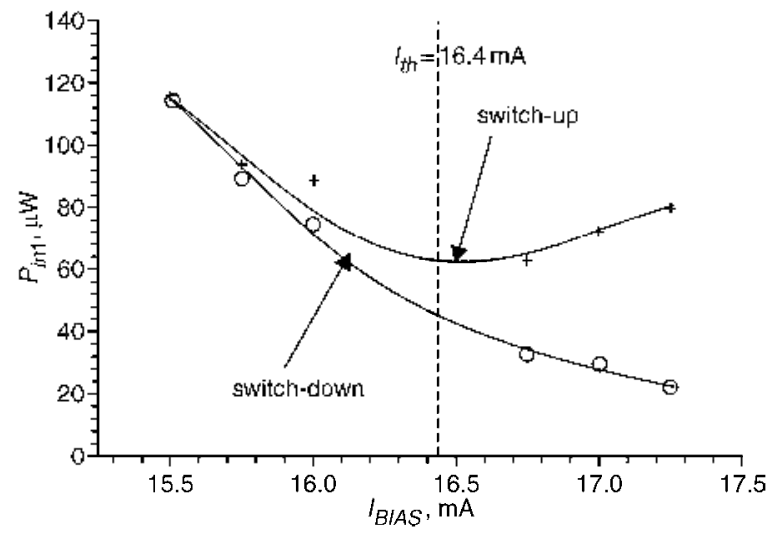

Fig. 4 Switch-up (crosses) and switch-down (circles) powers as a function of the applied bias current
DFBSOA and kept constant throughout the study, while the wavelength detuning of the input control signal (which is always detuned to the long-wavelength side of the resonant peak) varies from 0.2 to $0.3 \mathrm{~nm}$. Secondly, Figs. $7(a-d)$ show the same $\mathrm{I} / \mathrm{O}$ power relation as in Figs. $6(a-d)$, but now both input signals are detuned to the long-wavelength side of the resonant peak. The wavelength of the input control signal is now constant and detuned $0.25 \mathrm{~nm}$, while the wavelength of the input signal is varied and detuned, $0,0.07$ and $0.14 \mathrm{~nm}$ also to the long wavelength side of the resonant peak of the DFBSOA, resulting in a wavelength difference between both input signals of $0.25,0.18$ and $0.11 \mathrm{~nm}$ respectively, as seen in Figs. $7(a-d)$. For both cases, the main results are summarised in Table 3.

In both cases, keeping the wavelength of the input signal beam $\left(\lambda_{1}\right)$ fixed and keeping the wavelength of the input control signal $\left(\lambda_{2}\right)$ fixed, the higher the separation between both input signals, the greater the power needed for switching and the wider the hysteresis cycles. The effect of the wavelength separation on the bistable switching is, once again, the same as that observed when studying the influence of the wavelength detuning on the optical bistability in a DFBSOA with a single input beam $[20,21]$.

\section{Discussion}

In this work two-wavelength switching with a DFBSOA has been demonstrated. Using this feature, an optical signal might be placed in either of the two different wavelengths used in the experiment by choosing properly the power levels of the input signals. But to analyse the performance of an optical switch two main parameters have to be considered: power consumption and switching speed.

The studies carried out in this work show that bistable switching can be achieved with power requirements between 50 and $150 \mu \mathrm{W}$. However, higher on-off extinction ratios, wider bistable loops and lower input power requirements for switching are observed when the DFBSOA is biased with a current slightly above its threshold value. Nevertheless, as the bias current applied to the DFBSOA is increased to higher levels above 

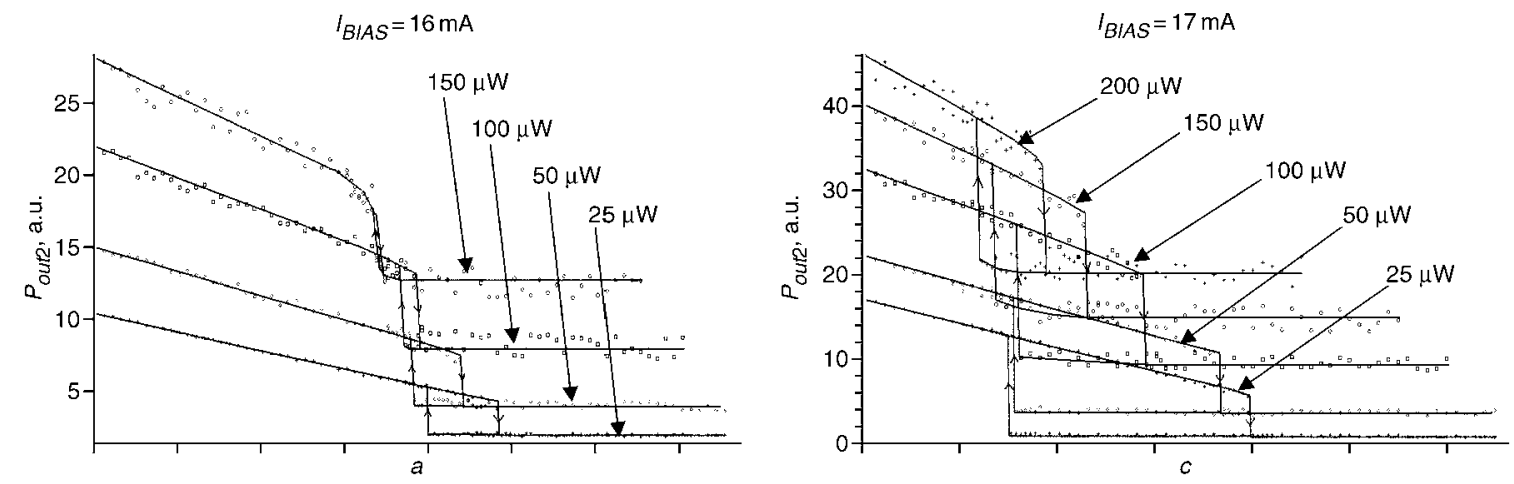

$\lambda_{1}-\lambda_{\mathrm{DFBSOA}}=0.25 \mathrm{~nm} ; \lambda_{2}=\lambda_{\mathrm{DFBSOA}}$
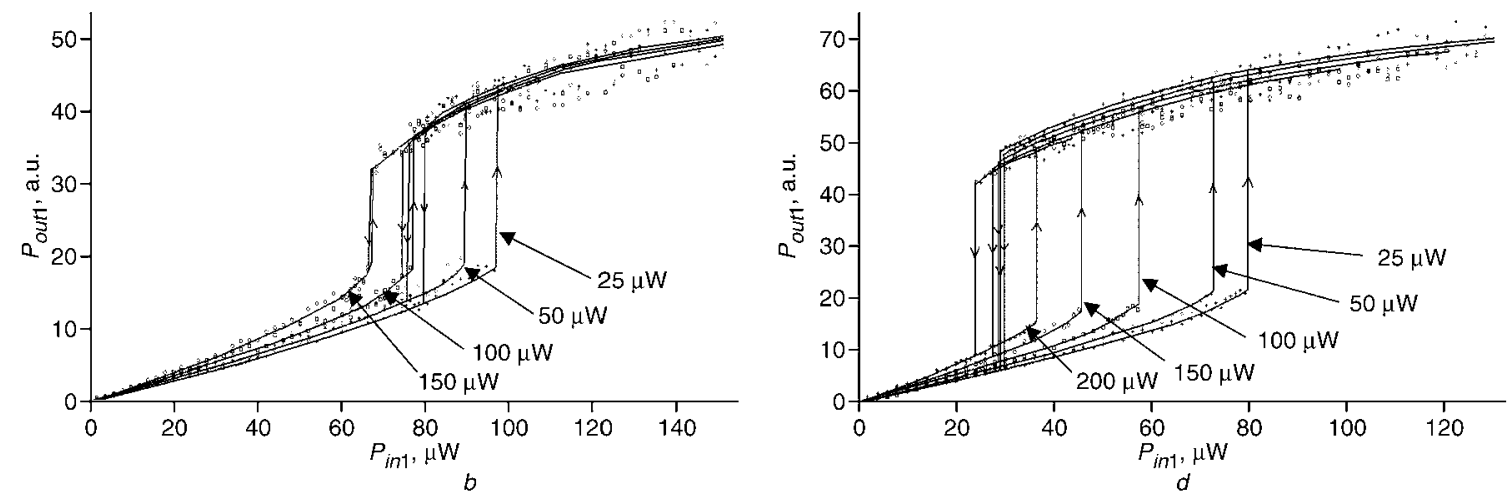

Fig. 5 Two-wavelength switching for different optical power levels of the input signal beam $\left(P_{\text {in } 2}\right)$

a $\mathrm{I} / \mathrm{O}$ power characteristic of the control signal

$b$ Signal beam versus input control for a bias current of $16 \mathrm{~mA}$

$c$ and $d$ Same results when the bias current is $17 \mathrm{~mA}$
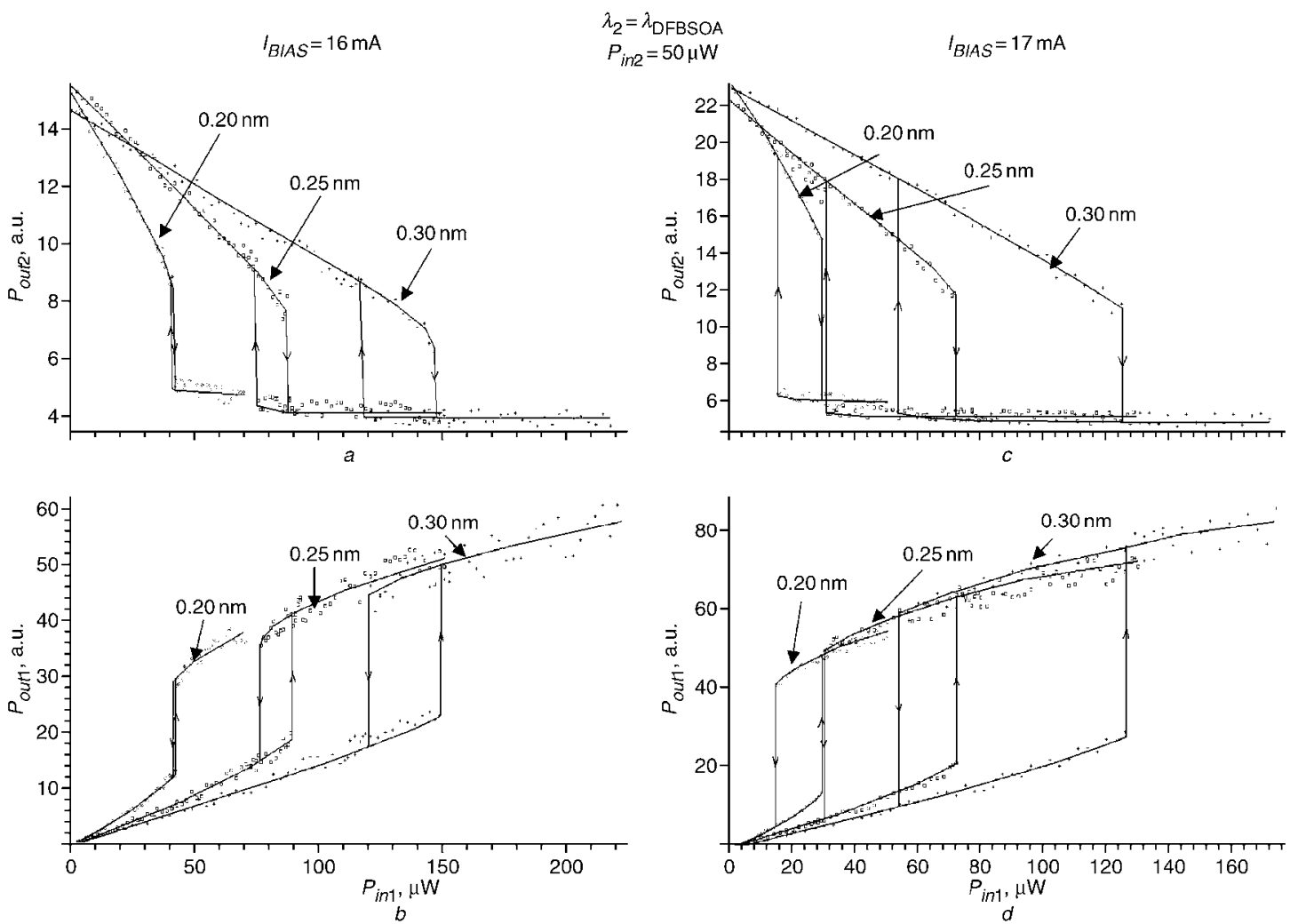

Fig. 6 Two-wavelength switching for distinct wavelength difference between both input signals when the wavelength of the input signal $\left(\lambda_{2}\right)$ is kept constant and equal to the resonant peak of the DFBSOA

a $\mathrm{I} / \mathrm{O}$ power characteristic of the control signal

$b$ Signal beam versus input control for a bias current of $16 \mathrm{~mA}$

$c$ and $d$ Same results when the bias current is $17 \mathrm{~mA}$ 

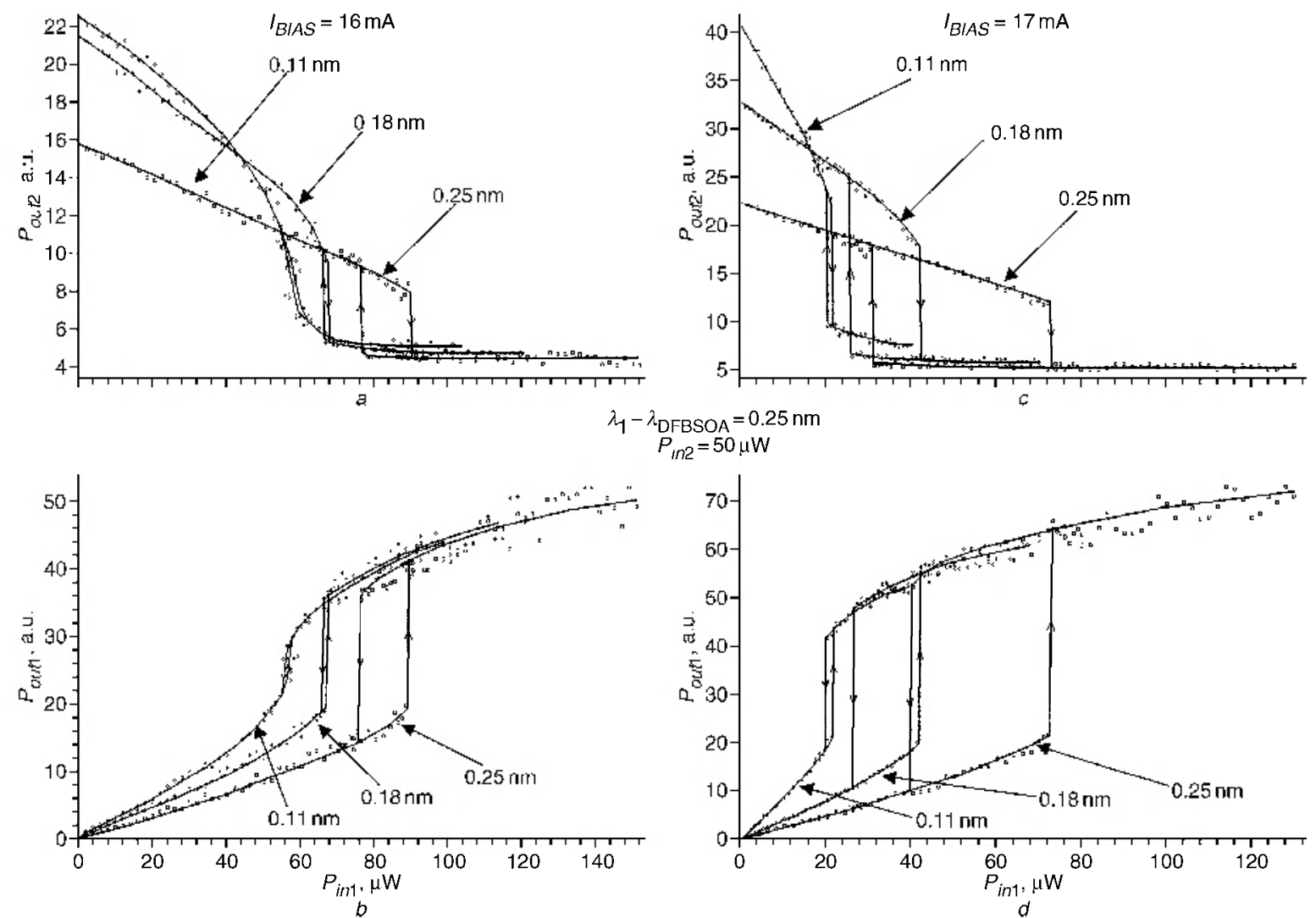

Fig. 7 Two-wavelength switching for distinct wavelength difference between both input signals when the wavelength of the control signal $\left(\lambda_{1}\right)$ is kept constant and detuned $0.25 \mathrm{~nm}$ to the long wavelength side of the resonant peak of the DFBSOA

a $\mathrm{I} / \mathrm{O}$ power characteristic of the control signal

$b$ Signal beam versus input control for a bias current of $16 \mathrm{~mA}$

$c$ and $d$ Same results when the bias current is $17 \mathrm{~mA}$

threshold, the power requirements for switching will exceed those required for the below threshold case. In terms of power consumption, the minimum requirements for switching operation will occur when the DFBSOA is operated slightly above threshold. Moreover, Hui et al. [29] reported that optical bistability in a DFBSOA is faster when the device is operated well above threshold, as the carrier dynamics are governed by the inverse of the relaxation oscillation and not by the effective carrier lifetime as in the case below threshold. Consequently, the two-wavelength switching should be faster above than below threshold.

In previous studies, two-wavelength switching with a FPSOA operated below threshold has been demonstrated at a bit rate of $800 \mathrm{Mb} / \mathrm{s}$ and power requirements below $100 \mu \mathrm{W}[11,12]$. Also, using a DFBSOA operated well-above threshold wavelength conversion and waveform reshaping was reported at a bit rate of $3 \mathrm{~Gb} / \mathrm{s}$ but now with a power requirement in the order of $\mathrm{mW}[9,10]$. Based on these results, it is straightforward to see that the increase of: the operating speed of the DFBSOA has an associated power penalty as the bias current applied to the DFBSOA is increased above threshold. The best compromise that can be achieved in the two-wavelength switching operation with a DFBSOA between the power and speed will happen when the device is operated slightly above threshold.

\section{Conclusions}

Two-wavelength switching operation between two optical signals at different wavelengths is demonstrated with a

Table 3: Study of the influence of the wavelength difference between both input signals in the two-wavelength switching with a DFBSOA

\begin{tabular}{|c|c|c|c|c|c|c|c|c|c|c|c|c|}
\hline Bias current $(\mathrm{mA})$ & 17 & & & & & & 16 & & & & & \\
\hline \multirow[t]{3}{*}{ Wavelength gap (nm) } & \multirow{2}{*}{\multicolumn{3}{|c|}{$\begin{array}{l}\lambda_{2}-\lambda_{1} \\
\left(\lambda_{2}-\lambda_{\mathrm{DFBSOA}}=0\right)\end{array}$}} & \multirow{2}{*}{\multicolumn{3}{|c|}{$\begin{array}{l}\lambda_{2}-\lambda_{1} \\
\left(\lambda_{1}-\lambda_{\text {DFBSOA }}=0.25 \mathrm{~nm}\right)\end{array}$}} & \multirow{2}{*}{\multicolumn{3}{|c|}{$\begin{array}{l}\lambda_{2}-\lambda_{1} \\
\left(\lambda_{2}-\lambda_{\mathrm{DFBSOA}}=0\right)\end{array}$}} & \multirow{2}{*}{\multicolumn{3}{|c|}{$\begin{array}{l}\lambda_{2}-\lambda_{1} \\
\left(\lambda_{1}-\lambda_{\mathrm{DFBSOA}}=0.25 \mathrm{~nm}\right)\end{array}$}} \\
\hline & & & & & & & & & & & & \\
\hline & 0.20 & 0.25 & 0.30 & 0.11 & 0.18 & 0.25 & 0.20 & 0.25 & 0.30 & 0.11 & 0.18 & 0.25 \\
\hline Switch-up power $\lambda_{1}(\mu W)$ & 30 & 73 & 129 & 22 & 42 & 73 & 42 & 89 & 150 & 55 & 66 & 89 \\
\hline Switch-down power $\lambda_{1}(\mu W)$ & 14 & 30 & 51 & 19 & 25 & 30 & 41 & 75 & 118 & 55 & 66 & 75 \\
\hline Hysteresis cycle $(\mu \mathrm{W})$ & 16 & 43 & 78 & 3 & 17 & 43 & 1 & 14 & 32 & 0 & 0 & 14 \\
\hline Minimum on-off ratio $\lambda_{1}$ & 3.57 & 2.97 & 2.70 & 2.04 & 2.73 & 2.97 & 2.35 & 2.05 & 2.04 & 1.26 & 1.83 & 2.05 \\
\hline Minimum on-off ratio $\lambda_{2}$ & 2.35 & 2.22 & 2.29 & 2.39 & 2.93 & 2.22 & 1.74 & 1.70 & 1.51 & 1.34 & 1.92 & 1.70 \\
\hline
\end{tabular}

The switching between states occur at equal power levels both in $\lambda_{1}$ and $\lambda_{2}$ 
$1.55 \mu \mathrm{m}$-DFBSOA. The two-wavelength switching mechanism is based in the reflective optical bistability occurring in a DFBSOA under external optical injection of two optical input signals. The two-wavelength switching properties have been analysed as the bias current applied to the DFBSOA is increased from below to above the threshold value and also for different levels of optical input power and initial wavelength detuning between the two optical input signals. Higher on-offiextinction ratios, wider bistable loops and lower input requirements for switching are assessed as the DFBSOA is operated slightly above threshold. Together with these results, the achievable switching speed increases as the bias current is incremented from below to above threshold which leads to the attainment of the best power/speed compromise in the switching operation of the DFBSOA when it is biased slightly above threshold. Under these conditions, alloptical two-wavelength switching between two signals at different wavelength, both in the $1550 \mathrm{~nm}$ range, could be performed with input power requirements below $100 \mu \mathrm{W}$ and switching speed in the order of $\mathrm{Gb} / \mathrm{s}$. Moreover, as clockwise and counterclockwise bistability is demonstrated in the operation of a DFBSOA under external injection of two optical signals, this could open the door for the development of AND, OR, NAND and NOR all-optical logic gates. In summary, the configuration reported in this work might be used for all-optical signal processing applications either for optical switching in optical telecommunication networks or for optical logic or optical memory applications in optical computing.

\section{Acknowledgments}

This work was supported in part by the Programa de Formación de Personal Investigador (FPI) de la Comunidad Autónoma de Madrid, CAM (Spain). The authors are grateful to an anonymous referee for constructive comments and suggesting the inclusion of Fig. 2.

\section{References}

1 Kawaguchi, H.: 'Bistabilities and nonlinearities in laser diodes' (Artech House, Norwood, MA, USA, 1994)

2 Sharfin, W.F., and Dagenais, M.: 'High contrast, $1.3 \mu \mathrm{m}$ optical AND gate with gain', Appl. Phvs. Lett., 1986, 48, (22), pp. 1510-1512

3 Wen, P., Sánchez, M.D., Gross, M., and Esener, S.: 'Vertical-cavity optical AND gate', Opt. Commun., 2003, 219, pp. 383-387

4 Hurtado-Villavieja, A., and Gonzalez-Marcos, A.: 'An all-optical programmable logic gate with $1550 \mathrm{~nm}$ laser diode amplifiers". Proc. Optics in Computing 2004, OiC2004, Engelberg, Switzerland, 21-23 April 2004, pp. 5-6

5 Inoue, K.: 'All-optical flip-flop operation in an optical bistable device using two lights of different frequencies', Opt. Lett., 1987, 12, pp. $918-920$

6 Maywar, D.N., Agrawal, G.P., and Nakano, Y.: 'All-optical hysteresis control by means of cross-phase modulation in semiconductor optical amplifiers', J. Opt. Soc. Am. B, 2001, 18, (7), pp. 1003-1013

7 Kim, Y.-I., Kim, J.H., Lee, S., Woo, D.H., Kim, S.H., and Yoon, T.-H.: 'Broad-band all-optical flip-flop based on optical bistability in an integrated SOA/DFB-SOA', IEEE Photonics Technol. Iett, 2004,16 , (2), pp. $398-400$
8 Nonaka, K., Noguchi, Y., Tsuda, H., and Kurokawa, T.: 'Digital signal regeneration with side-injection-light-controlled bistable laser diode as a wavelength converter, IEEE Photonics Technol. Lett., $1995,7,(1)$, pp. $139-141$

9 Inoue, K., and Yoshino, M.: 'Bistability and waveform reshaping in a DFB-LD with side-mode light injection', IEEE Photonics Technol. Lett., 1995, 7, (2), pp. 164-166

10 Inoue, K., and Oda, K.: "Noise suppression in wavelength conversion using a light-injected laser diode', IEEE Photonics Technol. Iett., $1995,7,(5)$, pp. $500-501$

11 Kawaguchi, H., Tani, H., and Inoue, K.: 'Optical bistability using a Fabry-Perot semiconductor-laser amplifier with two holding beams", Opt. Iett., 1987, 12, (7), pp. 513-515

12 Inoue, $\mathrm{K}$.: 'High speed all-optical gate switching experiment in a Fabry-Perot semiconductor laser amplifier', Electron. Lett., 1987, 23, (18), pp. 921-922

13 Okada, M., Kikuchi, H., Takizawa, K., and Fujikake, H.: 'Optical bistability and set-reset operation of a Fabry-Perot semiconductor laser amplifier with two detuned light injections', IEEE J. Quantum Electron., 1991, 27, (8), pp. 2003-2015

14 Grosskopf, G., Rudwig, R., Molt, R., Weber, H.G., and Zhang, S.B.: "Semiconductor-laser amplifier as an optically controlled switch", Appl. Phys. B, 1988, 45, (3), pp. 171-174

15 Maywar, D.N., Nakano, Y., and Agrawal, G.P.: '1.31-1.55 wavelength conversion by optically pumping a distributed feedback amplifier', IEEE Photonics Technol. Lett., 2000, 12, (7), pp. $858-860$

16 Maywar, D.N., and Agrawal, G.P.: 'Robust optical control of an optical-amplifier-based flip-flop', Opt. Express, 2000, 6, (3), pp. $75-80$

17 Otsuka, K., and Iwamura, H.: 'Analysis of a multistable semiconductor light amplifier', IEEE J. Quantum Electron., 1983, QE-19, (7), pp. 1184-1186

18 Otsuka, K., and Kobayashi, S.: 'Optical bistability and nonlinear resonance in a resonant-type semiconductor laser amplifier", Electron. Lett., 1983, 19, (7), pp. 262-263

19 Adams, M.J., Westlake, H.J., O’Mahony, M.J., and Henning, I.D.: 'A comparison of active and passive optical bistability in semiconductors', IEEE J. Quantum Electron., 1985, 21, (9), pp. 1498-1504

20 Zeqi Pan, Hongchin Lin, and Dagenais, M.: 'Switching power dependence on detuning and current in bistable diode laser amplifiers', Appl. Phys. Iett., 1991, 58, (7), pp. 687-689

21 Pakdeevanich, P., and Adams, M.J.: 'Measurements and modeling of reflective bistability in $1.55-\mu \mathrm{m}$ laser diode amplifiers", IEEE J. Quantum Electron., 1999, 35, (12), pp. 1894-1903

22 Kawaguchi, H., Inoue, K., Matsuoka, T., and Otsuka, K.: 'Bistable output characteristics in semiconductor laser injection locking', IEEE J. Quantum Electron., 1985, QE-21, (9), pp. $1314-1317$

23 Adams, M.J., and Wyatt, R.: 'Optical bistability in distributed feedback semiconductor laser amplifiers', IEE Proc. Pt. J. Optoelectron., 1987, 134, (1), pp. 58-63

24 Maywar, D.N., and Agrawal, G.P.: 'Transfer-matrix analysis of optical bistability in DFB semiconductor laser amplifiers with nonuniform gratings', IEEE J. Quantum Electron., 1997, 33, (11), pp. $2029-2037$

25 Sánchez, M., Wen, P., Gross, M., and Esener, S.: 'Nonlinear gain in vertical-cavity semiconductor optical amplifiers', IEEE Photonics Technol. Lett. 2003, 15, (4), pp. 507-509

26 Hurtado, A., Gonzalez-Marco, A., and Martin-Pereda, J.A.: 'Modeling reflective bistability in vertical-cavity semiconductor optical amplifiers", IEEE J. Quantum Electron, 2005, 41, (3), pp. 376-383

27 Adams, M.J.: 'Optical amplifier bistability on reflection', Opt. Quantum Electron, 1987, 19, S37-S45

28 Pakdeevanich, P.., and Adams, M.J.: 'Switching powers for optical bistability in a semiconductor laser above and below threshold", Opt. Commun, 2000, 176, pp. 195-198

29 Hui, R., Paradisi, A., Benedetto, S., and Montrosset, I.: 'Dynamics of optically switched bistable laser diodes in the injection-locked state", Opt. Lett. 1993, 18, (20), pp. 1733-1735 